\section{Rinossinusite fúngica em pacientes com infecção nasossinusal crônica}

\author{
Celso Dall'Igna ${ }^{1}$, Bruno Carlos Palombini ${ }^{2}$, \\ Fabio Anselmi, Elisabetb Araújó, \\ Daniela Pernigotti Dall'Igna ${ }^{5}$
}

\section{Fungal rhinosinusitis in patients with chronic sinusal disease}

Resumo / Summary

Palavras-chave: sinusite, micose, fungo, nariz. Key words: sinusitis, mycosis, fungus, nose.
$\mathbf{O}$ com infecção nasossinusal crônica. Nas últimas décadas houve aumento das infecções fúngicas, e a rinossinusite fúngica (RSF) tem sido mais freqüentemente diagnosticada. O conhecimento da flora fúngica, da sua prevalência e da apresentação sintomática em pacientes portadores de rinossinusite crônica (RSC) permitirá um melhor entendimento da doença, fato importante para a realização do diagnóstico, estabelecimento do tratamento e formulação do prognóstico. Forma de estudo: clínico retrospectivo com coorte transversal. Material e Método: 62 pacientes com diagnóstico de RSF foram selecionados entre 890 portadores de RSC submetidos à cirurgia endoscópica. Avaliou-se anamnese, exame otorrinolaringológico com videoendoscopia nasal, TC dos seios da face e exames microbiológicos e histopatológico. Resultados: A prevalência de RSF foi de 6,7\% em portadores de RSC submetidos à cirurgia endoscópica dos seios paranasais, e o tipo de fungo mais encontrado foi do gênero Aspergillus. Bola fúngica foi encontrada em mais da metade dos casos, e RSFA, em mais de um terço dos pacientes. Conclusões: A evolução sintomática após a cirurgia endoscópica foi mais favorável nos portadores de bola fúngica, que necessitaram menor número de reintervenções.

\begin{abstract}
A
im: Fungal rhinosinusitis in patients with chronic sinusal disease study. In the past decades, there has been an increase in fungal infections, and fungal rhinosinusitis (FRS) has been diagnosed more frequently. Knowing the fungal flora, its prevalence and symptomatic presentation in patients with chronic rhinosinusitis (CRS) will allow a better understanding of this disease, permitting a correct diagnosis, and treatment and formulating its prognosis. Study design: clinical retrospective with transversal cohort. Material and Method: 62 patients diagnosed with FRS were selected among 890 cases of CRS undergoing endoscopic surgery. We assessed clinical history otolaryngologic examination with nasal videoendoscopy, CT scan, microbiologic and histopatologic tests. Results: The prevalence of FRS was $6.7 \%$ and the fungal type most frequently found was the gender Aspergillus. Fungal ball was found in more than half the cases, and allergic fungal rhinosinusitis (AFRS) in more than one third of the patients. Evolution after endoscopic sinus surgery was more favorable in patients with fungus ball, with a minor number of recurrences. Conclusions: The symptomatic evolution following endoscopic surgery was more favorable in patients with fungal ball, who require a lower number of re-interventions.
\end{abstract}

\footnotetext{
${ }^{1}$ Doutor em Medicina pela UFRGS, Professor Adjunto de Otorrinolaringologia da UFRGS, Chefe do Serviço de ORL do Hospital de Clínicas de Porto Alegre. ${ }^{2}$ Professor Titular de Pneumologia da UFRGS, Chefe do Serviço de Pneumologia - Pavilhão Pereira Filho, Santa Casa de Misericórdia de Porto Alegre. ${ }^{3}$ Médico Otorrinolaringologista.

${ }^{4}$ Mestre e Doutor em Medicina. Professora de Pós-Graduação em Pneumologia da UFRGS. Médica Otorrinolaringologista.

${ }^{5}$ Médica Residente em Otorrinolaringologia da UFPR. Universidade Federal do Rio Grande do Sul.

Endereço para correspondência: Celso Dall'Igna - Rua Ramiro Barcelos 910/403 Moinhos de Ventos 90035-011 Porto Alegre, RS. Artigo recebido em 26 de maio de 2005. Artigo aceito em 5 de agosto de 2005.
} 


\section{INTRODUÇÃO}

Os fungos estão presentes na maior parte dos ecossistemas, existindo mais de 20.000 espécies já identificadas. Nas duas últimas décadas a medicina foi testemunha do aumento das infecções fúngicas em humanos, causadas por mais de 250 espécies diferentes, em decorrência do aumento do uso de drogas imunossupressoras para o tratamento do câncer e para o controle da rejeição de órgãos transplantados, das infecções pelo vírus da imunodeficiência humana (HIV), do diabetes melito, do uso de cateteres vasculares e urinários e do emprego de antibióticos de largo espectro. Desde uma comum candidíase até uma mortal aspergilose, as micoses estão freqüentemente presentes em todos os níveis da prática médica diária.

A rinossinusite fúngica (RSF) é referida na literatura médica há mais de dois séculos. Entretanto, somente nos últimos 25 anos vem recebendo a devida atenção, com o aumento da suspeição diagnóstica e melhora das técnicas laboratoriais de detecção dos fungos. Sua classificação, baseada na relação imunológica entre o fungo e seu hospedeiro e no grau de invasão da mucosa, é importante para a escolha de um tratamento efetivo e para o estabelecimento do prognóstico. O conhecimento do tipo de flora fúngica, da sua prevalência, da apresentação sintomática, dos aspectos do exame físico e dos exames subsidiários em pacientes portadores de rinossinusite crônica (RSC) permitirá um melhor entendimento da doença, alertando os médicos envolvidos com esses pacientes para o diagnóstico e tratamento adequado.

O presente estudo foi idealizado para que se venha a conhecer a prevalência de infecções por fungos em pacientes portadores de RSC submetidos a tratamento cirúrgico, sua classificação segundo o tipo de RSF, assim como sua apresentação clínica e sua evolução com o tratamento realizado.

\section{MATERIAL E MÉTODO}

\section{Delineamento do Estudo}

O presente estudo compreendeu uma coorte de pacientes portadores de RSC submetidos a tratamento cirúrgico dos seios da face pelo método endoscópico, no período compreendido entre janeiro de 1988 e dezembro de 2002 em Porto Alegre, RS, com diagnóstico de RSF, em que o fator em estudo foi a classificação dos pacientes, e o desfecho, a evolução de cada um deles.

\section{Critérios de inchusão e exchusão}

Foram avaliados 890 pacientes portadores de RSC submetidos a procedimento cirúrgico endoscópico dos seios da face e selecionados para estudo 62 com o diagnóstico de RSF, confirmado por exame micológico direto e/ou cultural e/ou por exame anatomopatológico.
Consideraram-se portadores de RSC pacientes com sinais e sintomas de inflamação nos seios paranasais que persistem por mais de 12 semanas, associada a alterações documentadas por técnicas de imagem após pelo menos 4 semanas de tratamento clínico adequado. ${ }^{1}$

Foram excluídos os pacientes que não concordaram em participar do estudo ou assinar o termo de consentimento informado, os que não tiveram um seguimento pósoperatório mínimo de 6 meses e os que não preencheram os critérios acima.

\section{Método de Coleta das Amostras}

Os pacientes tiveram as secreções do meato médio e ou do seio da face comprometido foram coletadas unilateralmente, sob visão endoscópica, com aspirador esterilizado em autoclave de $2 \mathrm{~mm}$ de diâmetro acoplado em recipiente de coleta Specimen Trap modelo 076-0490 (Sherwood Medical, St. Louis, EUA).

\section{Avaliação dos Pacientes}

Anammese - Compreendida história clínica geral e dirigida para a área otorrinolaringológica com a avaliação dos seguintes dados: obstrução nasal, rinorréia, prurido nasal, espirros, gota pós-nasal, epistaxe, alterações do olfato, queixas de orofaringe, cefaléia, rouquidão, tosse, asma e expectoração.

Exame Otorrinolaringológico - O exame incluía videoendoscopia nasal, realizada com endoscópio rígido de $4 \mathrm{~mm}$ e ângulo de $30^{\circ}$ e $0^{\circ}$ ou endoscópio flexível de $6 \mathrm{~mm}$, sob anestesia local, com algodão embebido com neotutocaína a $2 \%$, e vasoconstrição com oximetazolina quando necessário. Foram observados os seguintes dados: desvio do septo nasal, aspecto dos cornetos médio e inferior, coloração da mucosa, presença e tipo de secreção, polipose, degeneração polipóide e o estado do meato médio.

Estudo radiológico - Foi realizado TC dos seios da face, sem contraste intravenoso, nos planos axial e coronal, com o paciente em decúbito dorsal. Presença de microcalcificações e imagem de densidade metálica no interior do seio da face foram consideradas como sugestivas da presença de fungos. ${ }^{2,3}$

Exame microbiológico - As amostras clínicas foram coletadas durante o procedimento cirúrgico e colocadas em meio de transporte de Stuart (Starplex Scientific, Ontário, Canadá) para cultivo de microorganismos aeróbios, e em caldo de tioglicolato para cultivo de anaeróbios. O material foi encaminhado para análise no máximo 1 hora após a coleta.

Para a cultura aeróbia, o material era semeado em placas contendo o meio de ágar McConkey (Difco, Detroit, EUA ou Becton Dickinson, Maryland, EUA) e Tripticase Soy ágar (Difco, Detroit, EUA) enriquecido com 10\% de sangue de carneiro (ágar sangue) e incubado a $37^{\circ} \mathrm{C}$ por 24 horas. 
Não havendo crescimento bacteriano, os meios eram reincubados por mais de 24 horas antes de serem liberados como negativos.

O cultivo para germes anaeróbios foi realizado através de semeadura em ágar sangue de carneiro, tendo como base o ágar sangue de brucela (Difco, Detroit, EUA) e a bacteróide bile esculina ágar, com incubação por até 72 horas em atmosfera da anaerobiose proporcionada pelos sistemas Gaspak (Becton Dickinson, Maryland, EUA), Anaerocult (Merck SA, Brasil) ou Anaerobac (Probac, São Paulo, Brasil). O caldo tioglicolato era utilizado como back up para semeadura de anaeróbios em caso de suspeita de presença de germes na amostra (estimada pelo método de Gram) e ausência de crescimento nas placas. Após o isolamento e a confirmação de se tratar de germe anaeróbio, a identificação do microorganismo era feita com a utilização do sistema API para germes anaeróbios (Bio Merieux, França).

A análise micológica foi realizada através do exame direto do material, entre lâmina e lamínula, e a cultura do material em meio de Sabouraud com ou sem cloranfenicol e ciclohexamida. A incubação foi a $25^{\circ}-35^{\circ} \mathrm{C}$, e as culturas foram observadas até 20 dias para liberação como negativas para fungos. A identificação dos fungos e leveduras foi feita a partir da morfologia microscópica e da utilização de kit comercial para identificação de leveduras (Sistema API, BioMerieux, França), respectivamente.

Exame Histopatológico - As amostras da mucosa do seio envolvido, de acordo com os achados histopatológicos, foram classificadas como:

a. inflamação crônica inespecífica (edema com espaçamento da lâmina própria da mucosa, infiltrado linfoplasmocitário e fibrose perivascular);

b. inflamação crônica supurativa (características anteriores com infiltrado neutrocitário);

c. inflamação crônica alérgica (inflamação crônica com infiltrado eosinofílico);

d. inflamação crônica com invasão fúngica (visualização de hifas filamentosas e segmentadas com coloração pelo método PAS e Grocot).

\section{Classificação dos Pacientes}

Os pacientes foram classificados quanto ao tipo de RSF, de acordo com os achados cirúrgicos e histopatológicos, como portadores de bola fúngica, infecção saprófita, RSF invasiva, RSFA e RSF indolente, conforme os critérios adotados por Ferguson ${ }^{4}$ :

a. bola fúngica: caracterizada por um emaranhado de hifas no interior do seio da face, sem invasão tecidual e com mínima reação inflamatória da mucosa;

b. infestação saprófita: presença assintomática de fungos visíveis em crostas mucosas no nariz ou no seio de face, sem invasão da mucosa; c. rinossinusite fúngica invasiva: infecção fúngica com invasão tecidual ao exame histopatológico, com ou sem invasão vascular;

d. rinossinusite fúngica alérgica: presença de muco com numerosos eosinófilos, cristais de Charcot-Leyden, raras hifas e reação inflamatória da mucosa, sem invasão por fungos;

e. rinossinusite fúngica indolente ou crônica invasiva: presença de fungos com invasão vascular ausente ou mínima.

\section{Seguimento dos pacientes}

O seguimento dos pacientes foi realizado pelos autores com anamnese, exame otorrinolaringológico completo e exame endoscópico nasal. O acompanhamento foi realizado por um período mínimo de seis meses.

\section{Análise Estatística}

Para a comparação de proporções foi usado o teste do qui-quadrado, com correção de Yates quando o número de graus de liberdade fosse igual a um, e o teste exato de Fischer se o número esperado para determinada característica fosse inferior a 5 pacientes.

Os sintomas foram comparados usando-se uma escala de escores com o teste t para amostras emparelhadas (dados paramétricos) e o teste T de Wilcoxon (dados nãoparamétricos para amostras emparelhadas), e ANOVA (Analyses of Variance) para medidas repetidas para a comparação de médias.

Para $\pm=0,05$ e $2=0,20$ e desejando identificar diferenças de exposição entre casos e controles de pelo menos $20 \%$ a 25\%, estimou-se um tamanho amostral mínimo de 60 pacientes por grupo (total $=120$ ). O erro aceitável foi de $5 \%(\mathrm{p}<0,05)$.

O projeto teve seu protocolo aprovado pelo Comitê de Ética do Hospital de Clínicas de Porto Alegre.

\section{RESULTADOS}

De um total de 890 procedimentos cirúrgicos nasossinusais realizados foram selecionados os casos em que foi detectada a presença de fungos identificados em exames laboratoriais, que são o objeto do presente estudo.

Foram analisados os dados de $62(6,7 \%)$ pacientes com idade média de 40,6 anos (variando de 8 a 81 anos, mediana de 45,4 anos), sendo 25 do sexo masculino e 37 do sexo feminino, sem haver diferença significativa $(\mathrm{p}=$ 0,13 , teste $t$ de Student) entre eles. Apenas 1 paciente era cor negra, sendo os demais da cor branca.

Os sintomas e sinais encontrados encontram-se resumidos na Tabela 1. O tempo de duração dos sintomas variou de 3 meses a mais de 5 anos.

As comorbidades mais freqüentemente encontradas foram rinite em 33 pacientes (53,2\%), asma brônquica em 
21 (33,8\%), hipertensão arterial sistêmica em 16 (25,8\%) e intolerância à aspirina em 14 (22,5\%). Com incidência menor aparecem broncopneumonias de repetição (7 pacientes), bronquiectasias (6 pacientes), hepatite (4 pacientes), transplantes de órgãos e imunodeficiência primária (3 pacientes), diabetes melito e leucemia ( 2 pacientes), e casos isolados de fibrose cística, tuberculose e tumor de hipófise. Quanto ao uso prévio de medicação, 59 pacientes $(95,1 \%)$ haviam utilizado antibiótico de largo espectro no mês anterior à cirurgia, $37(59,6 \%)$ eram usuários de corticosteróide nasal tópico, 25 (40,3\%) utilizavam corticosteróide oral e 6 (9,7\%) já haviam recebido algum tipo de medicação imunossupressora que não corticosteróide.

Apenas 11 pacientes $(18,6 \%)$ haviam se submetido anteriormente à cirurgia nasossinusal: 3 a polipectomia endoscópica, 4 a sinusotomia, 1 a septoplastia, 1 a rinoplastia, 1 a septoplastia e polipectomia e 1 a turbinectomia e sinusotomia.

Os achados à endoscopia nasal foram: secreções nasais em 57 pacientes $(91,9 \%)$, sendo $29(46,7 \%)$ amareladas, 23 (25\%) esverdeadas, 4 tinham cor marrom e 1 de cor preta; obstrução do meato médio em $47(75,8)$, polipose em 25 (40,3\%), sendo bilateral em $10(16,1 \%)$ e unilateral em 15 (24,1\%), hipertrofia de conchas inferiores em 17 $(27,4 \%)$ e hipertrofia de adenóides em 3 (4,8\%).

Todos os pacientes apresentavam algum tipo de velamento à TC dos seios da face, estando os demais achados listados na Tabela 2.

O exame cultural micológico do material obtido durante a cirurgia mostrou os fungos do gênero Aspergillus como o mais freqüente, seguido pelos gêneros Candida e Penicilium (Tabela 3).

O exame bacteriológico indicou infecção bacteriana associada em 47 pacientes, havendo o crescimento de $S$. aureus em 15 casos (31,9\%), P. aeruginosa em 10 (21,2\%) e Haemophillus sp. em 4 (8,5\%). Em 10 pacientes (16,9\%) não houve crescimento bacteriano e em 2 o exame não foi realizado.

Polipectomia foi realizada em 23 pacientes, sendo em 6 unilateral e em 17 bilateral.

O exame histopatológico definitivo mostrou inflamação crônica inespecífica em 25 casos (40,3\%), inflamação crônica alérgica em $21(33,8 \%)$, inflamação crônica supurativa em 12 (19,3\%) e invasão fúngica em 4 (6,4\%). Quanto à classificação da RSF observou-se bola fúngica em 33 pacientes (53,2\%), RSFA em 24 (38,7\%) e RSF fúngica indolente em 3 (4,8\%). Em 2 pacientes os fungos foram considerados saprófitas. Não houve nenhum caso de rinossinusite fúngica invasiva (Tabela 4).

Entre os casos classificados como bola fúngica, obstrução nasal estava presente em 30 (90,9\%) deles, secreção nasal posterior em $29(87,8 \%)$ e de corticosteróides tópicos em apenas $9(27,3)$. Tosse foi queixa em $19(57,5 \%)$, intolerância à aspirina aparecem somente em $1(3,5 \%)$ pacien- te e $6(18,2 \%)$ tiveram pneumonia de repetição. Pólipo foi evidenciado em apenas $2(7,1 \%)$ pacientes, e obstrução do meato médio em 7 (21,2\%).

Os pacientes com RSFA apresentavam, em sua totalidade, obstrução nasal, secreção nasal posterior e uso prévio de corticosteróides tópicos e antibióticos sistêmicos. Tosse

Tabela 1. Apresentação clínica pré-operatória dos pacientes em estudo.

\begin{tabular}{lcc}
\hline Sinais e Sintomas & $\mathrm{n}$ & $\%$ \\
\hline obstrução nasal & 57 & 92 \\
secreção nasal & 55 & 89 \\
gota pós-nasal & 51 & 82 \\
tosse & 43 & 69 \\
rinite alérgica & 30 & 48 \\
alterações do olfato & 21 & 34 \\
asma & 21 & 34 \\
irritação na garganta & 20 & 32 \\
intolerância a aspirina & 14 & 22 \\
fadiga & 14 & 22 \\
dor facial & 9 & 15 \\
\hline
\end{tabular}

Tabela 2. Achados tomográficos.

\begin{tabular}{lcc}
\hline & $\mathrm{n}$ & $\%$ \\
\hline Velamento & 62 & 100,0 \\
Obstrução de meato médio & 49 & 79,0 \\
Espessamento ou esclerose óssea & 34 & 54,8 \\
Microcalcificações & 28 & 47,4 \\
Imagem densidade metálica & 18 & 29,0 \\
Erosão óssea & 16 & 25,4 \\
Expansão do seio & 11 & 17,7 \\
\hline
\end{tabular}

Tabela 3. Tipos de fungos identificados ao exame cultural micológico.

\begin{tabular}{lcc}
\hline & $\mathrm{n}$ & $\%$ \\
\hline Aspergillus $s p$. & 30 & 48,3 \\
Candida $s p$ & 11 & 17,7 \\
Penicillium & 5 & 8,0 \\
hifas septadas e ramificadas & 5 & 8,0 \\
Alternaria $s p$. & 3 & 4,8 \\
outros & 8 & 12,8 \\
\hline
\end{tabular}

Tabela 4. Tipos de rinossinusite fúngica.

\begin{tabular}{lcc}
\hline & $\mathrm{n}$ & $\%$ \\
\hline Bola fúngica & 33 & 53,2 \\
RSF alérgica & 24 & 38,7 \\
RSF indolente & 3 & 4,8 \\
Infecção saprófita & 2 & 3,2 \\
RSF invasiva & 0 & - \\
\hline Total & 62 & 100.0 \\
\hline
\end{tabular}


foi queixa em 14 (58,3\%), 12 (50\%) referiram intolerância à aspirina e apenas $5(20,1 \%)$ tiveram pneumonia prévia. Os achados cirúrgicos evidenciaram pólipos em 22 pacientes (91.6\%), obstrução do meato médio em $21(87,5 \%)$ e microcalcificações em 20 (81,8\%) dos casos. O tipo de fungo mais freqüente foi o Aspergillus (8 casos).

A Tabela 5 mostra a comparação dos achados préoperatórios entre estes 2 grupos.

Não houve diferença estatística ao serem comparados a idade média ( $p=0,13$, teste t de Student) e o sexo do grupo com diagnóstico de RSFA com os de bola fúngica. Os sintomas pré-operatórios foram analisados estatisticamente após serem colocados em uma escala e comparados globalmente entre os dois diagnósticos. O escore dos grupos, quando comparado, utilizando-se o teste t para amostras independentes, não evidenciou diferença entre eles $(p=0,715$; IC 95\%: -1,11 a 1,61).

Por outro lado, uso de corticosteróides tópicos, história de intolerância prévia à aspirina e asma brônquica, quando analisados individualmente, foram os únicos que mostraram diferença estatística na comparação dos sintomas préoperatórios entre os dois grupos, sendo mais freqüentes nos pacientes com diagnóstico de RSFA. A presença de pólipos ao exame clínico pré-operatório em pacientes portadores de RSFA $(78,2 \%)$ foi significativamente maior ( $p<0.001$, teste $t$ de Student) do que a dos com o diagnóstico de bola fúngica $(6,0 \%)$.

Comparando-se os estudos radiográficos com tomografia computadorizada dos dois grupos, o achado de obstrução do meato médio não mostrou diferença significativa (teste do Qui-quadrado). Os demais achados, como a presença de erosão óssea, foram encontrados em 12 pacientes $(50 \%)$ com RSFA e em $4(12,1 \%)$ com bola fúngica $(\mathrm{p}<0.001)$, microcalcificações em $19(79,1 \%)$ e $10(30,3 \%)$ $(\mathrm{p}<0.001)$, e espessamento da mucosa em $8(33,3 \%)$ e 2 $(6,1 \%)$ pacientes $(\mathrm{p}=0,02)$, respectivamente, evidenciando uma diferença significativa entre os grupos.

Quanto ao tipo de fungo encontrado nas culturas, não houve diferença estatística entre os grupos.
A Tabela 6 mostra a comparação dos achados cirúrgicos entre os pacientes com o diagnóstico de bola fúngica e os com RSFA.

O tempo de acompanhamento pós-operatório até o momento da análise dos dados foi em média de 19,4 meses, com desvio padrão de 6,3 meses. Vinte pacientes $(32,2 \%)$ foram acompanhados de 6 meses a 1 ano, 14 (22,6\%), de 1 a 2 anos 6 de 3 a 4 anos (9,7\%), 7(11,2\%) de 4 a 5 anos e $15(24,2 \%)$ tiveram mais de 5 anos de acompanhamento pós-operatório.

Tabela 5. Achados pré-operatórios segundo o tipo de rinossinusite fúngica.

\begin{tabular}{lccc}
\hline Sintoma & \multicolumn{2}{c}{ Tipo de RFS } & Significância \\
& RSFA & Bola Fúngica & \\
\hline Obstrução nasal & 100 & 91 & $\mathrm{NS}^{*}$ \\
Secreção posterior & 100 & 88 & $\mathrm{NS}^{*}$ \\
Corticosteróide tópico & 100 & 29 & $\mathrm{p}<0,001^{* *}$ \\
Antibiótico sistêmico & 100 & 94 & $\mathrm{NS}^{*}$ \\
Rinite alérgica & 81 & 36 & $\mathrm{NS}^{*}$ \\
Asma & 77 & 11 & $\mathrm{p}<0,001^{* *}$ \\
Tosse & 58 & 57 & $\mathrm{NS}^{*}$ \\
Intolerância AAS & 50 & 3 & $\mathrm{p}<0,02^{\star *}$ \\
Broncopeumonia repetição & 20 & 18 & $\mathrm{NS}^{*}$ \\
\hline $\mathrm{p}=$ Valores-p de Pearson para o teste do Qui-quadrado \\
*NS - não significativo ( $\mathrm{p}>0,05$ ) \\
** Correção de Yates do Qui-quadrado
\end{tabular}

Tabela 6. Achados trans-operatórios nos casos de rinossinusite fúngica alérgica e de bola fúngica.

\begin{tabular}{lccc}
\hline Sintoma & \multicolumn{2}{c}{ Tipo de RFS } & Significâ ncia \\
& RSFA & Bola fúngica & \\
\hline Obstrução meato médio & 88 & 24 & $\mathrm{p}<0,001$ \\
Microcalcificação & 79 & 33 & $\mathrm{p}<0,02^{* *}$ \\
Pólipos & 91 & 6 & $\mathrm{p}<0,001^{*}$ \\
\hline
\end{tabular}

$\mathrm{p}=$ Valores- $\mathrm{p}$ de Pearson para o teste do qui-quadrado

* Teste exato de Fisher

** Correção de Yates do Qui-quadrado

Tabela 7. Avaliação dos sintomas no período pós-operatório

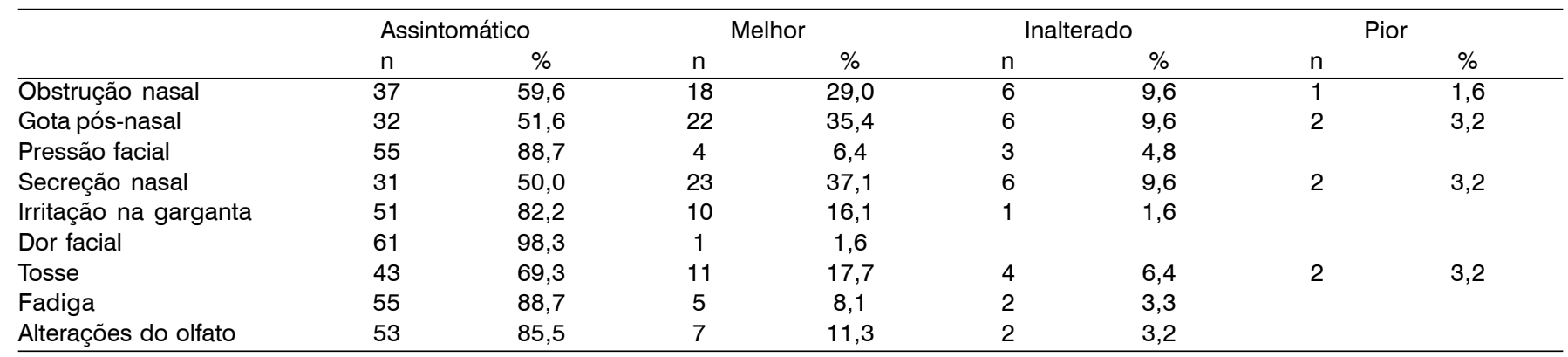


A avaliação geral dos sintomas no pós-operatório de todos os pacientes pode ser vista na Tabela 7 .

Na Tabela 8 podemos evidenciar a melhora sintomática significativa das principais queixas dos pacientes portadores de RSFA e bola fúngica, comparando sua presença no pré e no pós-operatório.

Quando comparamos os sintomas dos dois grupos após o procedimento cirúrgico, usando a mesma escala de escores, evidenciamos que os sintomas nos pacientes com bola fúngica são significativamente menores dos que nos com RSFA, seja qual for o tipo teste realizado. O teste paramétrico (teste t para amostras emparelhadas) mostrou p < 0,0001 com IC 95\% de 2,38 a 3,89; no teste T de Wilcoxon (não-paramétrico para amostras emparelhadas) encontramos $\mathrm{p}<0,0001$, valor que se repetiu na ANOVA para medidas repetidas.

Dezesseis pacientes $(25,8 \%)$ apresentaram recorrência e 14 deles necessitaram de reintervenção cirúrgica: 9 pela recidiva da polipose nasal, 4 pela recidiva fúngica e 1 por rinorréia purulenta não responsiva a tratamento farmacológico. O intervalo entre o procedimento cirúrgico primário e a reintervenção variou de 10 meses a 11 anos.

Onze (45,8\%) pacientes com RSFA necessitaram de reintervenção cirúrgica (figura 7): 10 (41,6\%) devido à recidiva da doença e da polipose e em um caso em razão do aparecimento de doença contra-lateral. Apenas 3 pacientes (9\%) com bola fúngica tiveram necessidade de submeter-se à nova cirurgia. O número de reintervenções foi significativamente maior $(\mathrm{p}<0,002)$ nos pacientes com RSFA do que nos portadores de bola fúngica.

Quanto ao uso de medicamentos no período pósoperatório, 7 pacientes (11,2\%) (3 com infecção indolente e 4 com RSFA) receberam antifúngico por via oral, 56 (90,3\%) receberam antibióticos e 55 (88,7\%) receberam lavagem nasal com solução salina. Para os pacientes com RSFA, corticosteróides por via oral foi prescrito para 83\% deles corticosteróide nasal tópico para 87,5\%.

Tabela 8. Evolução dos sintomas dos pacientes com bola fúngica e rinossinusite fúngica alérgica após a intervenção cirúrgica.

\begin{tabular}{lcccc}
\hline & RSFA & & \multicolumn{2}{c}{ Bola Fúngica } \\
& antes & após & antes & após \\
\hline Obstução nasal* $^{*}$ & 100 & 23 & 90 & 4 \\
Gota pós-nasal* $^{*}$ & 100 & 23 & 84 & 10 \\
Secreção nasal* $^{*}$ & 95 & 23 & 97 & 10 \\
Irritação garganta* $^{*}$ & 77 & 14 & 58 & 13 \\
Tosse* $_{\text {Alterações olfato* }}$ & 68 & 9 & 13 & - \\
\hline
\end{tabular}

\section{DISCUSSÃO}

Aproximadamente 300 espécies de fungos já foram documentadas como causadores de doença em humanos ${ }^{5} \mathrm{e}$ 90\% das infecções são atribuídas a poucas dezenas de espécies. ${ }^{6}$ A maioria dos fungos é exógena, existindo no solo, na água e em restos orgânicos. As micoses de maior incidência são causadas por poucos fungos que fazem parte da flora normal, como a candidíase, ou são altamente adaptados para sobreviverem no organismo humano, como as dermatofitoses. ${ }^{6}$

A RSF, apesar de ser pouco freqüente, é uma doença que produz significativa morbidade, podendo levar à morte. Seus sintomas são facilmente sobrepostos a outras situações, sendo difícil seu reconhecimento pelo médico e, portanto, seu diagnóstico. A avaliação e o manejo destes quadros podem requerer a participação de vários especialistas, incluindo otorrinolaringologista, infectologista, microbiologista e patologista, propiciando diagnóstico e tratamento adequados, prevenindo seqüelas permanentes e diminuindo a mortalidade.

A incidência e a prevalência das infecções fúngicas estão em crescimento com diversificação dos organismos patogênicos, em decorrência do aumento dos fatores de risco, como atopia na RSFA, cetoacidose diabética na mucormicose e uso de corticosteróides na candidíase. ${ }^{7}$ Colabora também para esse crescimento o comprometimento da imunidade celular nos pacientes com SIDA e nos pacientes que cada vez mais utilizam drogas imunossupressoras ou citotóxicas, seja para o combate a neoplasias, seja pela participação em programas de transplante de órgãos, cada vez mais freqüentes na prática médica diária. ${ }^{8}$

O diagnóstico da RSF inicialmente requer um alto grau de suspeição por parte do médico, pois a história clínica e o exame físico, por si só, raramente são conclusivos.

Schell ${ }^{9}$ considera importante que a presença do fungo no meato médio ou no seio da face seja identificada para que posteriormente possamos pensar em responder à difícil diferenciação entre colonização e patogenicidade, e que os métodos laboratoriais a serem adotados sejam os mais simples possíveis, para se tornarem acessíveis à rotina médica diária.

A verdadeira incidência da RSF, especialmente da RSFA entre os pacientes portadores de RSC, permanece desconhecida. ${ }^{4}$ Demonstramos uma prevalência de $6,7 \%$ de culturas positivas para fungos na série de pacientes portadores de RSC submetidos a procedimento cirúrgico, valor maior que o encontrado por Lessa et al. ${ }^{10}(\mathrm{p}<0,05$, teste do qui quadrado) em um estudo realizado em hospital público na cidade de São Paulo, que mostrou a incidência de fungos em 31 pacientes $(4,4 \%)$ dos 706 casos com RSC operados entre os anos 1995 e 2000. Esta diferença de prevalência pode ser explicada por nosso interesse maior na identificação dos fungos, objetivo primordial do estudo. Katzenstein et al., ${ }^{11}$ em 1983, na descrição da RSFA, encontraram uma 
incidência de 6,2\%; Kupfenberg e Bent ${ }^{12}$ estimam que o fungo é a causa de 5\% a 7\% dos casos de RSC que necessitam de tratamento cirúrgico; e Yoon et al., ${ }^{3}$ na Coréia do Sul, avaliando de 510 pacientes com RSC submetidos à cirurgia do seio maxilar encontraram $39(7,6 \%)$ casos de RSF comprovados hitopatologicamente.

Quaraishi e Ramadan ${ }^{13}$ estimam a prevalência de fungos em 7\%; Shubert, ${ }^{14}$ em uma revisão bibliográfica de estudos retrospectivos, encontrou valores entre 5 e 10\%, coincidindo com o registrado por Houser e Corey $^{15}$ para pacientes portadores de RSC com indicação cirúrgica.

Vennevald et al., ${ }^{16}$ entretanto, obtiveram cultura positiva para fungos em $25,7 \%$ de 117 pacientes imunossuprimidos com RSC não-invasiva submetidos à cirurgia endoscópica dos seios da face, fato que pode ser explicado pelo estado imunológico dos pacientes. Lebowitz et al., ${ }^{17}$ estudando 45 pacientes com RSC submetidos a procedimento cirúrgico endoscópico, isolaram fungos em 56\% deles, usando métodos laboratoriais tradicionais, idênticos aos empregados rotineiramente no processamento de amostras de secreção pulmonar.

Por serem os fungos onipresentes, a contaminação de amostras para cultura pode ocorrer facilmente durante a coleta ou o processamento laboratorial. Consideramos que a simples identificação do fungo não necessariamente comprova sua patogenicidade, e o diagnóstico de uma infecção micótica somente pode ser realizado quando analisamos em conjunto os dados da cultura, os achados cirúrgicos e o resultado do exame anatomopatológico.

Marple e Mabry ${ }^{5}$ destacam o papel dos fungos no desenvolvimento e na perpetuação da infecção na via aérea superior, e observaram existirem na literatura fortes evidências das formas alérgica e não alérgica da inflamação fúngica não-invasiva. Ainda não sabemos se tais formas de inflamação são independentes ou inter-relacionadas.

Os dados publicados em diversos estudos são extremamente variados quando analisamos as espécies de fungos identificadas em paciente com RSF. Existem um grande número de espécies e uma importante variação geográfica regional da sua prevalência. Os gêneros mais citados são Aspergillus, Alternatia, Candida, Penicilium, e Fusarium. ${ }^{4,5,7}$

Encontramos espécies de Aspergillus em mais da metade das amostras, dado também descrito por Manning, ${ }^{2}$ que considera este gênero como predominante nos casos de RSF não-invasiva, fato também constatado por Donald ${ }^{18}$ e por Ruppa el al. ${ }^{19}$ Vennewald et al. ${ }^{16}$ encontraram Aspergillus sp. em $23(67,4 \%)$ pacientes de uma série de 34 portadores de RSF sem alteração no sistema imunológico, e Kupfenberg ${ }^{20}$ em 19 (41,3\%) de uma série com 49 pacientes com essa mesma patologia.

A incidência de um determinado gênero depende muito das condições geográficas e climáticas de onde habita o paciente, pois a presença de um determinado fungo no ambiente está relacionada com as condições ambientais de temperatura e umidade relativa do ar. $^{4}$

Quando são identificados fungos como agentes etiológicos das rinossinusites, é importante classificarmos o tipo de RSF para se poder planejar um tratamento adequado e determinar um prognóstico, que depende muito mais da resposta do hospedeiro do que do tipo particular de fungo que causa a infecção. Inicialmente a doença pode-se dividir em dois tipos básicos: invasivo ou não-invasivo, com base na penetração do fungo na mucosa. As RSFs nãoinvasivas são diagnosticadas como bola fúngica, infestação saprófita ou RSFA. ${ }^{4}$ Dentre elas, a RSFA é a que mais provoca controvérsia na literatura. Seus critérios diagnósticos foram propostos inicialmente por Katzenstein, ${ }^{11}$ como a presença de fungos sem invasão da mucosa, muco espesso, inflamação eosinofílica e cristais de Charcot-Leyden.

$\mathrm{Na}$ apresentação clínica dos pacientes com diagnóstico de RSF, os sintomas predominantes que encontramos foram obstrução nasal (92\%), secreção nasal (88,7\%), gota pós-nasal (82,2\%) e tosse (69,35\%), comuns a todos os doentes com RSC, portanto sem muita importância na suspeita da presença de fungos. A comorbidade mais freqüente em nossa série foi a rinite alérgica (53,2\%), praticamente a mesma incidência (62\%) assinalada por Kalinger ${ }^{21}$ em um estudo de 200 pacientes consecutivos com RSC que compareceram à consulta com alergista ou imunologista. A freqüência de asma brônquica $(38,2 \%)$ é semelhante à encontrada por Manning. ${ }^{2}$

Após o procedimento cirúrgico, os pacientes foram classificados quanto ao tipo de RSF de acordo com os critérios adotados por Ferguson, ${ }^{4}$ baseados nos achados cirúrgicos, microbiológicos e histopatológicos.

Nenhuma ocorrência de RSF invasiva foi identificada em nossa série. A RSF invasiva é uma situação que compromete pessoas com doença graves associadas, e seu diagnóstico dificilmente é feito em pacientes não hospitalizados. ${ }^{4,8}$ Ocorre sempre em pacientes imunocomprometidos, seja em portadores de SIDA ou nos que recebem corticosteróides ou drogas imunossupressoras para o tratamento de neoplasias ou após transplante de órgãos. Diabéticos em cetoacidose são de alto risco para doença invasiva causada por fungos da classe dos Zigomicetes, que preferem ambientes ácidos com alta concentração de glicose. A ausência de RSF invasiva em nossa série pode ser explicada pela origem dos pacientes, todos ambulatoriais, não portadores de doenças graves.

Baseados nestes achados decidimos concentrar o estudo nos dois grupos mais prevalentes, bola fúngica e RSFA, comparando os achados clínicos e cirúrgicos e sua evolução após o tratamento.

Ao compararmos a idade entre os dois grupos não encontramos diferença entre eles, apesar de a bola fúngica ser citada na literatura como uma situação em que as pessoas de idade mais avançada são mais afetadas., ${ }^{4,22}$ 
Os achados pré-operatórios dos pacientes com diagnóstico de bola fúngica, quando comparados aos com RSFA, não evidenciaram diferença significativa quanto a secreção e obstrução nasal, uso de antibióticos sistêmicos, história de rinite alérgica e broncopneumonias de repetição. Os portadores de RSFA apresentaram um número significativamente maior de asma brônquica, intolerância à aspirina e uso de corticosteróides tópicos, fatos que podem ser explicados pela presença de atopia nestes pacientes. Ferguson, ${ }^{22} \mathrm{em}$ uma revisão da literatura, também não encontrou diferenças entre os sintomas dos pacientes com bola fúngica quando comparados com os portadores de outros tipos de RSF.

Já a presença de pólipos foi estatisticamente maior nos casos de RSFA, dados compatíveis com os de autores como Houser e Corey, ${ }^{15}$ que encontraram pólipos em todos os pacientes com RSFA, e os de Ferguson ${ }^{22}$, que os encontrou em 10\% dos portadores de bola fúngica. Portadores de RSFA também tiveram erosão óssea e espessamento da mucosa significativamente maiores. Houser e Corey, ${ }^{15}$ descrevem erosão óssea em apenas $20 \%$ dos casos em uma na revisão da literatura.

Após serem cotejados individualmente, os achados pré e pós-operatórios sofreram tratamento estatístico e foram colocados em uma escala de valores, possibilitando uma comparação global da sintomatologia entre os dois grupos. Esta comparação global dos sintomas estudados não mostrou diferença entre os mesmos, fato também referido por Ferguson, ${ }^{4}$ Houser e Corey ${ }^{15}$ e Manning. ${ }^{7}$

Entretanto, quando estes sintomas foram comparados individualmente, os portadores de RSFA apresentaram mais freqüentemente intolerância à aspirina, asma brônquica, presença de polipose nasal e uso anterior de corticosteróide tópico. Estes quatro fatores, assim como a RSFA, são reconhecidamente relacionados à atopia, característica que pode explicar tal diferença.

A grande freqüência de polipose nos pacientes portadores de RSFA é plenamente compatível com os achados publicados na literatura, sendo sua presença freqüentemente citada como critério diagnóstico. Houser e Corey ${ }^{15}$ consideram a incidência de pólipos de praticamente 100\%. Kupfenberg e Bent, ${ }^{12}$ e Manning ${ }^{2}$ encontraram pólipos em todos os pacientes de suas séries, submetidos a tratamento cirúrgico.

Nos pacientes com diagnóstico de bola fúngica, a presença de polipose em 2 (6,1\%) é semelhante ao encontrado por Ferguson ${ }^{22}$ (10\%) em uma revisão bibliográfica de 158 casos.

Na avaliação sintomática após o procedimento cirúrgico, observamos uma melhora de todos os itens avaliados em mais de $90 \%$ dos pacientes, comprovando a eficiência e o benefício da cirurgia, semelhantes aos resultados obtidos em pacientes operados por RSC. ${ }^{23,24}$

Usando a mesma escala de valores dos sintomas, observamos uma melhora sintomática significativamente mai- or nos portadores de bola fúngica, que tiveram também menos recidivas e necessitaram de um menor número de reintervenções, quando comparados com os de RSFA. Estes resultados evidenciam o caráter recidivante e de maior gravidade da RSFA.

Para os portadores de bola fúngica o índice de recidiva (9\%) é semelhante ao encontrado por Ferguson, ${ }^{22}$ estimado em revisão da literatura entre 4\% e 10\%. Manning ${ }^{7}$ e Kupfenberg ${ }^{20}$ consideram que as recidivas de bola fúngica são raras.

O número de recidivas nos portadores de RSFA foi bem maior, sendo que 45,8\% deles necessitaram de reintervenção cirúrgica, acentuando o caráter crônico e recidivante da doença e seu difícil controle. O sucesso do tratamento da RSFA depende de três etapas: efetuar debridamento cirúrgico para remover os antígenos fúngicos, a mucina alérgica e a mucosa polipóide doente; prevenir a recorrência do crescimento fúngico e modificar a resposta imunológica do hospedeiro ao antígeno. Como nem sempre conseguimos um controle adequado da sintomatologia alérgica, podemos esperar um seguimento pós-operatório com um índice maior de recidivas.

Podemos constatar na literatura uma grande controvérsia sobre o real papel do fungo na RSC e concordamos com grande parte dos autores ${ }^{4,7,9,11,17}$ que consideram que a simples identificação do fungo não é suficiente para o diagnóstico, devendo este ser realizado em conjunto com os achados clínicos cirúrgicos e anatomopatológicos.

Existem poucas dúvidas sobre o fato de os fungos ou seus componentes protéicos podem estimular o trato respiratório através de mecanismos alérgicos mediados por IgE. A literatura oferece muitas evidências que explicam as formas alérgicas e não-alérgicas das infecções fúngicas não-invasivas. Devemos estabelecer no futuro o quanto estas formas estão interrelacionadas ou são independentes entre si.

A compreensão destes mecanismos imunológicos e da maneira como poderemos intervir sobre eles aumentará a efetividade dos diagnósticos e dos tratamentos, diminuído a recorrência da doença, principalmente nos casos de RSFA.

A infecção por fungos é um problema emergente na clínica médica diária, tendo sua prevalência aumentado devido ao grande número de doenças crônicas resultantes do aumento da idade média da população e/ou decorrentes de situações patológicas que requerem o uso de medicamentos que alteram o sistema imunológico.

Em nosso estudo, procuramos estabelecer a prevalência de infecções fúngicas em pacientes imunocompetentes, assim como o tipo de apresentação clínica da doença. O conhecimento destes fatos tem importância na suspeita clínica e na abordagem investigativa dos pacientes otorrinolaringológicos portadores de RSC em nossa clínica diária. 


\section{CONCLUSÕES}

A prevalência de RSF foi de $6,7 \%$ em pacientes portadores de RSC submetidos à cirurgia endoscópica dos seios paranasais, tendo predominado o gênero Aspergillus, havendo também quantidade significativa dos gêneros Candida, Peniciliume Alternaria.

O tipo de RSF mais freqüente foi bola fúngica em mais da metade dos casos, seguido pela RSFA, presente em mais de um terço dos pacientes. Os diagnósticos de RSF indolente e de infecção saprófita foram pouco freqüentes. A evolução após a cirurgia endoscópica foi mais favorável nos portadores de bola fúngica, que necessitaram um menor número de reintervenções.

Sugerimos que, no futuro, novos estudos venham a ser desenvolvidos, complementando o aqui levado em efeito, principalmente incluindo pacientes imunossuprimidos e com doença invasiva, que não fizeram parte de nossa investigação.

O aumento do nosso conhecimento e a compreensão sobre a doença fúngica nos fará melhorar a suspeita clínica, a acuidade do diagnóstico e a efetividade dos tratamentos, proporcionando um prognóstico melhor a nossos pacientes.

\section{REFERÊNCIAS BIBLIOGRÁFICAS}

1. International Rhinosinusitis Advisory Board. Infectious rhinosinusitis in adults: classification, etiology and management. Ear Nose Throat J 1997; 76:1-19.

2. Manning SC, Merkel M, Kriesel K, Vuitch F, Marple B. Computed tomography and magnetic resonance diagnosis of allergical fungal sinusitis. Laryngoscope 1997; 107:170-6.

3. Yoon JH, Na DG, Byun HS, et al. Calcification in chronic maxillary sinusitis: comparison of CT findings with histopathologic results. Am J Neuroradiol 1999; 20:571-4.

4. Ferguson BJ. Definitions of fungal rhinosinusitis. Otolaryngol Clin North Am 2000; 33:227-35.
5. Marple BF, Mabry RL. The role of fungus in chronic rhinosinusitis. Curr Opin Otolaryngol Head Neck Surg 2002; 10: 207-12.

6. Mitchel TG. Overview of basic medical mycology. Otolaryngol Clin North Am 2000 33:237-49.

7. Manning SC. Fungal Sinusitis. In: Rhinology and Sinus Disease. Schaefer SD, ed. St Louis: Mosby, 1977; 99-104.

8. Hunt SM, Miyamoto C, Cornelius RS, Tami TA. Invasive fungal sinusitis in the acquired immunodeficiency syndrome. Otolaryngol Clin North Am 2000; 33:335-47.

9. Schell WA. Histopathology of fungal rhinosinusitis. Otolaryngol Clin North Am 2000; 33:251-75.

10. Lessa MM, Marcondes RA, Goto EY, Romano OF, Voegels RL, Butugan O. Cirurgia endoscópica endonasal: experiência em 706 casos. Rev Bras Otorrinol 2001; 67:515-7.

11. Katzenstein AL, Sale SR, Greenberger PA. Allergic aspergillus sinusitis: a newly recognized form of sinusitis. J Allergy Clin Immunol 198; 372:89-93.

12. Kupferberg SB, Bent JP. Prognosis for allergic fungal sinusitis. Otolaryngol Head Neck Surg 1997; 117:35-41.

13. Quraishi H, Ramadan HH. Endoscopic treatment of allergic fungal sinusitis. Otolaryngol Head Neck Surg 1977; 117:29-34.

14. Schubert MS. Medical treatment of allergic fungal sinusitis. Ann Allergy Asthma Immunol 2000; 85:90-101.

15. Houser SM, Corey JP. Allergic fungal rhinosinusitis. Otolaryngol Clin North Am 2000; 33:399-417.

16. Vennewald I, Henker M, Klemm E, Seebcher C. Fungal colonization of the paranasal sinuses. Mycoses 1999; 42:33-6.

17. Lebowitz RA, Waltzman MN, Jacobs JB. Pearlman A, Tierno PM. Isolation of fungi by standard laboratory methods in patients with chronic rhinosinusitis. Laryngoscope 2002; 112:2189-91.

18. Donald PJ. Fungal infection of the sinuses. In: Donald PJ, Gluckman JL, Rice DH, eds. The sinuses. New York: Raven Press 1994; 271-85.

19. Rupa V, Jacob M, Mathews MS. Increasing diagnostic yeld in allergic fungal sinusitis. J Laryngol Otol 2001; 115:636-8.

20. Kupfenberg SB. Fungal sinusitis: current trends in diagnosis and treatment. In: Medscape Respiratory Care 2000; 4:45-51.

21. Kaliner MA, Osguthorpe JD, Fireman P. Rhinosinusitis: bench to bedside. Otolaryngol Head Neck Surg, 1997; 116:S1-S19.

22. Ferguson BJ. Fungus balls of the paranasal sinus. Otolaryngol Clin North Am 2000; 33:389-97.

23. Marple B, Newcomer M, Schwande N, Mabry R. Natural history of allergic fungal rhinosinusitis: a 4- to 10-year follow-up. Otolaryngol. Head Neck Surg 2002; 127:361-6.

24. Kennedy DW. Sinus disease: guide to first-line management. Deerfield Beach: Health Communications; 1994. p.1-44. 